\title{
P01-41
}

\section{TEMPERAMENT AND CHARACTER INVENTORY DIMENSIONS IN ALCOHOL DEPENDENCY}

F. Karadag ${ }^{1}$, O. Saatcioglu ${ }^{2}$ I. Ure ${ }^{1}$, A. Ayhan ${ }^{1}$, N. Tellioglu' ${ }^{1}$, F. Ozdemir ${ }^{3}$, F. Akdeniz ${ }^{3}$, A.E. Tezcan ${ }^{1}$

${ }^{1}$ Psychiatry Department, Maltepe University Medical Faculty, ${ }^{2}$ Psychiatry Department, Bakirkoy Professor Mazhar Osman Uzman Hospital for Psychiatric and Neurological Diseases, Istanbul, ${ }^{3}$ Affective Disorders Unit, Ege University Medical Faculty Psychiatry Department, Izmir, Turkey

Aim: The aim of this study is to evaluate the differences in dimensions of temperament between alcohol dependent inpatients and healthy control subjects.

Method: The study is consisted of 77 alcohol-dependent patients according to the Diagnostic and Statistical Manual of Mental Disorders, 4th Edition and 80 healthy control subjects. Cloninger's Temperament and Character Inventory (TCI), Temperament Evaluation of Memphis, Pisa, Paris and San Diego Autoquestionnaire (TEMPS-A) scale and semi structured sociodemographic form were applied to the subjects after detoxification period.

Results: Age and education level were similar rates in both alcohol dependent (mean age $=43.7 \pm 10.1$ and education level= $9.0 \pm$ 4.2) and healthy control subjects (mean age: $42.5 \pm 11.8$ and education level: $10.2 \pm 3.8$ ). Alcohol-dependent patients were characterized by higher Novelty-Seeking $(p=0.006)$ and Harm Avoidance $(p=0.002)$ than non-psychiatric control subjects.Also, subjects suffering from Alcohol-dependence exhibited significantly more depressive $(p=0.003)$, anxious $(p=0.001)$, irritable $(p=0.004)$ and cyclothymic $(p=0.001)$ temperament. Novelty-Seeking [95\% C.I $=1.08(1.07-1.16)]$ and cyclothymic temperament $[95 \%$ C.I $=$ $1.13(1.03-1.23)]$ were predictors of alcohol dependency.

Conclusion: Novelty seeking and harm avoidance were significantly higher in the patients than in the healthy subjects. NoveltySeeking and cyclothymic temperament were predictors for alcohol dependency. It seems to be important to consider TCl dimensions and affective temperaments in planning the treatment and maybe in preventing of alcohol dependence. 